\title{
Molekularbiologie in der Viszeralchirurgie - prädiktive Diagnostik hereditärer Tumoren
}

\author{
Gesprächsleiter: H. K. Schackert, Dresden \\ Teilnehmer: $\quad$ W. Friedl, Bonn E. Holinski-Feder, München B. Irrgang, Dresden \\ G. Möslein, Düsseldorf St. Pistorius, Dresden J. Rüschoff, Kassel \\ H.-D. Saeger, Dresden
}

\section{Einleitung}

Neue Techniken der Molekularbiologie und deren konsequente Anwendung in der Klinik bahnten den Weg für die prädiktive molekulargenetische Diagnostik maligner Tumoren. Hochpenetrante Keimbahnmutationen, die zur familiären Häufung von Tumoren führen, werden in zunehmendem Maße und mit unterschiedlichem Erfolg bei bereits erkrankten Indexpersonen von betroffenen Familien identifiziert. Dies erlaubt die Prädiktion des individuellen Erkrankungsrisikos bei den Mitgliedern der Familie und den konsequenten Einschluß in oder Ausschluß aus dem strikten klinischen Überwachungsprogramm. Prädiktion eines Ereignisses mit hoher, jedoch nicht absoluter Präzision ist speziell in der Medizin mit zahlreichen Risiken und daraus resultierenden Fragen verbunden, denen sich die behandelnden Ärzte stellen müssen. Die molekulare Medizin basiert auf neuen Erkenntnissen, die derzeit exponentiell zunehmen, und der Zusammenarbeit verschiedener medizinischer und biologischer Fachrichtungen in hohem Maße.

Die vorgezeichneten Weiterentwicklungen der molekularen Grundlagenforschung läßt erwarten, daß zunehmend komplexere Zusammenhänge zwischen Genotyp und Phänotyp gefunden werden. Diese Annahme stützt sich auf die Prognose, daß das menschliche Genomprojekt in 3-5 Jahren alle Gene des menschlichen Genoms identifiziert und sequenziert haben wird. Viele Befunde deuten darauf hin, daß neben den hereditären Tumorerkrankungen auch die sogenannten sporadischen Tumoren eine genetische Basis haben. Vermutlich bestimmen zahlreiche Genvarianten zusammen mit exogenen Faktoren das individuelle Krankheitsrisiko eines Menschen. Diese Kenntnisse mit allen daraus resultierenden diagnostischen und therapeutischen Vor- und Nachteilen werden die molekulare Medizin der Zukunft bestimmen. Am Beispiel vererbter kolorektaler Karzinomsyndrome diskutieren Wissenschaftler und Kliniker aus den eng miteinander kooperierenden Fachbereichen Molekulargenetik, Humangenetik, Pathologie, Epidemiologie, Ethik und Chirurgie die derzeitigen Möglichkeiten und Grenzen der prädiktiven Diagnostik und geben einen vorsichtigen Ausblick in die Zukunft der molekularen Medizin.

\section{KARGER}

Fax +497614520714

E-mailkargergmbh@aol.com

www.karger.com
(C) 1999 S. Karger GmbH, Freiburg

Accessible online at:

http://BioMedNet.com/karger

\section{Frage: In welchen Bereichen der Viszeralchirurgie hat die prädiktive Diagnostik Bedeutung?}

Friedl: Die prädiktive Diagnostik ist vor allem von Bedeutung für die Früherkennung von Anlageträgern für erbliche Tumordispositionserkrankungen wie z. B. die familiäre adenomatöse Polyposis (FAP), das erbliche kolorektale Karzinom ohne Polyposis (HNPCC), das familiäre Mamma-/Ovarialkarzinom, multiple endokrine Neoplasie (MEN-I), Magen- und Nierenzellkarzinom. Die prädiktive Diagnostik ist insbesondere wichtig für die Erkennung derjenigen Personen aus Familien mit erblichen Tumorerkrankungen, die nicht Anlageträger sind, denn nur für diesen Personenkreis, bei dem die in der Familie bekannte Tumordisposition ausgeschlossen wird, ist die prädiktive Diagnostik tatsächlich relevant.

Möslein: In der Viszeralchirurgie zeichnet sich in den letzten Jahren ein völlig neues Indikationsspektrum für operative Eingriffe ab. Dieser Wandel ist in der prädiktiven Diagnostik hereditärer Prädispositionen für Karzinomerkrankungen begründet. Seit kurzem ist die Entfernung makroskopisch gesunder Organe, basierend auf dem molekulargenetischen Nachweis einer krankheitsverursachenden Mutation bei manchen Syndromen, indiziert. Heute spielt die prädiktive Diagnostik bereits eine Rolle bei MEN und HNPCC. Der Nachweis einer krankheitsrelevanten Mutation im RET-Protoonkogen führt zu der Indikationstellung einer prophylaktischen Thyreoidektomie im Vorschulalter. Bei Nachweis einer Mutation in den Mismatch-Reparatur-Genen ist eine prophylaktische Entfernung des Kolorektums zu diskutieren. Die Indikationsstellung zu der prophylaktischen Entfernung eines Organs, das noch keine Krankheitsmerkmale aufweist, hängt von der Penetranz des entsprechenden Gens und der Heterogenität der Organmanifestation $\mathrm{ab}$.

Rüschoff: Aus der Sicht des Pathologen stellt die molekulare prädiktive Diagnostik eine wesentliche Erweiterung des diagnostischen Spektrums, insbesondere im Bereich der Onkologie, dar. Traditionell werden auch heute noch Ausmaß und Art des chirurgi- 
schen Vorgehens nach Tumortyp, Tumorstadium und Malignitätsgrad (Typing, Grading, Staging) ausgerichtet. Künftig werden sich aber Therapieentscheidungen auch an den Ergebnissen der molekularen prädiktiven Analytik orientieren.

Saeger/Pistorius: Der Hauptschwerpunkt liegt hierbei zweifellos bei der prädiktiven Diagnostik hereditärer Tumorerkrankungen, d.h. insbesondere bei solchen Erkrankungen, die mit hochpenetranten Keimbahnmutationen assoziiert sind. Dies betrifft in erster Linie die verschiedenen Formen der hereditären kolorektalen Neoplasien (HNPCC, FAP), jedoch auch das familiäre medulläre Schilddrüsenkarzinom (FMTC, MEN-II) und ferner das familiäre Mammakarzinom.

\section{Frage: Welche Vorteile hat die molekulare Diagno- stik im Vergleich zur klinischen Diagnostik bei here- ditären Tumorsyndromen?}

Friedl: Die meisten hereditären Tumorsyndrome sind autosomaldominant erblich, d. h. daß bereits eine Mutation in einem der beiden Kopien des betreffenden Gens zu einer erhöhten Tumorneigung führt. Personen mit einem erhöhten Risiko für Tumoren bedürfen entsprechend dem betreffenden Krankheitsbild einer engmaschigen klinischen Vorsorge. Aufgrund des autosomal-dominanten Erbgangs sind alle Kinder eines Anlageträgers mit einer Wahrscheinlichkeit von 50\% ebenfalls Träger der Mutation. Mit klinischen Methoden kann man Anlageträger meist erst dann erkennen, wenn die betreffende Erkrankung schon Symptome zeigt - häufig ist zu diesem Zeitpunkt der Tumor bereits fortgeschritten.

Der «Vorteil» der molekulargenetischen Diagnostik besteht folglich darin, daß Anlageträger bereits identifiziert werden können, bevor Symptome der Erkrankung auftreten. Man kann daher die erforderlichen klinischen Vorsorgeuntersuchungen auf die tatsächlichen Anlageträger beschränken. Dies bedeutet einen großen Vorteil für die «Nichtträger» der Mutation, kann aber auch zu vielschichtigen psychosozialen Problemen in den Familien führen (siehe auch 6. Frage).

Holinski: Hier sind im wesentlichen drei Punkte zu nennen: 1. Durch den Nachweis einer genetischen Veränderung können die Risikopersonen einer Familie gezielt einer intensivierten Vorsorge zugeführt werden. 2. In manchen Fällen ist die klinische, differentialdiagnostische Abgrenzung hereditärer Tumorsyndrome schwierig. Eine kleine Anzahl kolorektaler Polypen können im Rahmen einer attenuierten FAP oder eines HNPCC-Syndroms auftreten. Beide Tumorsyndrome sind mit völlig unterschiedlichen anderen Neoplasien assoziiert und bedürfen somit eines anderen Vor- und Nachsorgeprogramms. In diesem Fall ist das besonders für das Endometriumkarzinom eklatant. Bei der FAP besteht für diese Tumorerkrankung kein erhöhtes Risiko, handelt es sich jedoch um ein HNPCC-Syndrom, muß für Frauen ein 30\%iges Risiko angenommen werden. 3. Das molekulargenetische Untersuchungser- gebnis wird im Sinne einer Genotyp-Phänotyp-Korrelation zunehmend an Bedeutung in der Therapie gewinnen.

Möslein: Der wesentliche Vorteil liegt in der Möglichkeit, nichtbetroffene Riskopersonen zu identifizieren und aus der spezifischen Vorsorge entlassen zu können. Sie sind somit von der Angst und Sorge eines erhöhten Karzinomrisikos befreit. Dies betrifft auch die nachfolgenden Generationen. Für Genträger ergibt sich die Bestätigung des auf der Familienanamnese begründeten Verdachts eines hohen Karzinomrisikos. Gefragt ist eine umfassende Betreuung eben jener Personen, die sowohl die medizinischen Probleme der Vorsorge und Früherkennung als auch das Auffangen psychologischer Probleme beinhaltet. Das Wissen um die Genträgerschaft und die somit frühe Kenntnis des hohen Risikos kann eine effektive Vorsorge überzeugend einleiten, mit einer zu postulierenden Prognoseverbesserung.

Rüschoff: Jeder klinisch-diagnostisch tätige Pathologe stellt täglich zahlreiche Tumordiagnosen. Angaben seitens der Klinik zur Familienanamnese sind dabei (noch!) die Ausnahme. Wir konnten z. B. für das erbliche HNPCC-assoziierte Dickdarmkarzinom zeigen, daß bereits das histologische Bild erste Hinweise auf eine mögliche erbliche Disposition geben kann. Da gerade für die Abklärung des HNPCC-Syndroms einfache molekulare Screening-Tests zur Verfügung stehen, die am Tumor durchgeführt werden, kann der Pathologe wesentlich zur Erfassung gerade des HNPCC-Syndroms beitragen.

Saeger/Pistorius: Hier sind mehrere Vorteile zu nennen: 1. Es handelt sich bei der molekularen Diagnostik um ein nichtinvasives Verfahren, sieht man von der notwendigen Blutentnahme ab. 2. Das Ergebnis bietet der molekularen Diagnostik durch die Identifizierung von Genträgern und Nichtgenträgern die Möglichkeit der gezielten klinischen Vorsorge in den betroffenen Familien mit der Konsequenz einer verbesserten Prognose durch Früherkennung einerseits und der Erübrigung unnötiger klinischer Untersuchungen andererseits. Dies wird zukünftig auch unter dem ökonomischen Aspekt eine wachsende Bedeutung erlangen. 3. Die molekulare Diagnostik gestattet uns durch die Charakterisierung des zu erwartenden Krankheitsbildes, beispielsweise der Tumorentwicklung des Patienten, eine individuell gestaltete Optimierung notwendiger operativer Therapien.

\section{Frage: Wo sind die Grenzen der prädiktiven mole- kularen Diagnostik?}

Friedl: Die Mutationen in den Genen, die zu hereditären Tumorsyndromen führen, sind - mit wenigen Ausnahmen - über weite Bereiche der betreffenden Gene verstreut (z. T. sind auch mehrere teilweise noch nicht bekannte Gene involviert), und selbst unter Einsatz aller heute verfügbaren molekulargenetischen Methoden kann nicht in jedem Fall die der Erkrankung zugrundeliegende Mutation festgestellt werden. Deshalb kann bei einer Risikoper- 
son eine Mutation nur dann ausgeschlossen werden, wenn die in der Familie vorhandene Mutation identifiziert wurde. Aus obengenannten Gründen sollte die molekulargenetische Untersuchung nur für individuelle Familien mit erhöhtem Tumorrisiko angeboten werden und nicht als Bevölkerungs-Screening.

Holinski: Prädiktive Diagnostik ist zur gezielten Erfassung von Risikopersonen in belasteten Familien hilfreich. Zwei Punkte sollten jedoch berücksichtigt werden: 1. Eine prädiktive molekulargenetische Testung sollte nur angeboten werden, wenn die bei einem betroffenen Familienmitglied krankheitsverursachende genetische Veränderung (Mutation) bekannt ist. Hierfür gibt es vier Gründe: (a) Familiäre Tumorsyndrome sind heterogen, was in der prädiktiven Situation ohne Kenntnis der Mutation in der Familie die Analyse mehrerer Gene bedeuten würde. Dies ist derzeit noch mit einem unverhältnismäßigen Aufwand verbunden, vor allem vor dem Hintergrund, daß wir (b) für die meisten Tumorsyndrome noch gar nicht alle Gene kennen und diese somit auch nicht analysieren können. (c) In manchen Familien ist eine eindeutige Zuordnung zu einem Tumorsyndrom nicht möglich; dies erhöht die Anzahl der zu analysierenden Gene, soweit man sie kennt, und damit die diagnostische Unschärfe. (d) Das A-priori-Risiko für einen Ratsuchenden mit einem erstgradig verwandten Betroffenen beträgt $50 \%$, diese Anlagen ebenfalls geerbt zu haben. Wenn man bei einer prädiktiven Diagnostik keine genetische Veränderung findet, kann es sein, daß die Mutation nicht vererbt wurde, im falschen Gen gesucht wurde, die Mutation in einem bislang unbekannten Gen lokalisiert ist und zuletzt keine der zur Verfügung stehenden Analysemethoden eine 100\%ige Sensitivität hat. 2. Die prädiktive Diagnostik sollte nur empfohlen werden, wenn auch ein wirkungsvolles klinisches Vorsorgeprogramm angeboten werden kann. In Familien mit Multitumorerkrankungen, wie z. B. beim Li-FraumeniSyndrom, sollte man sich das Angebot einer prädiktiven Diagnostik gut überlegen, denn zum einen ist nicht bei allen Tumorerkrankungen eine Früherkennung möglich bzw. mit einer besseren Prognose assoziiert (wie z. B. bei Tumoren des Zentralnervensystems), und zum anderen ist ein Vorsorgeprogramm, bei dem mehr oder weniger alle Organe berücksichtigt werden müssen, nicht zumutbar. Unsere Erfahrung hat gezeigt, daß auch bei klar umschriebenen Tumorsyndromen, wie z.B. dem erblichen Mammakarzinom, die prädiktive Mitteilung einer Anlageträgerschaft als ausgesprochen traumatisierend erlebt wird. Auch wenn viele Ratsuchende vor der Testung aufgrund ihrer Familienanamnese davon ausgehen, daß sie die Anlage geerbt haben, ist es doch ein Unterschied, dieses definitiv zu wissen, auch wenn in diesem Fall Hilfe angeboten werden kann. Die Situation wird mit unserer häufigsten Anfrage charakterisiert: «Warum können Sie bei mir, auch wenn ich gesund bin, keinen Gentest machen? Mein Arzt schickt mich.»

Möslein: Die Grenzen der prädiktiven molekularen Diagnostik machen sich vor allem darin klinisch bemerkbar, daß ein Genträgerstatus für ein bestimmtes Syndrom zwar eindeutig nachgewiesen werden kann, aber eine Vorhersage bezüglich des weiteren klinischen Verlaufs nicht möglich ist. Als Beispiel hierfür sei die familieninterne Heterogenität der Krankheitsausprägung herangezogen. Beim HNPCC können die Organmanifestation und das Diagnosealter bei erkrankten Personen mit der gleichen krankheitsverursachenden Mutation erheblich variieren. Es muß davon ausgegangen werden, daß hier sogenannte Disease-modifiers eine erhebliche Bedeutung haben. Ob es sich um genetische Faktoren handelt, die den Effekt der Mismatch-repair-Mutation ganz oder teilweise aufheben, oder aber um den Beweis des Einflusses exogener Faktoren, ist unbekannt.

Rüschoff: Bei allem Enthusiasmus für neue Methoden und Wege in der Medizin sollte der klinische Befund, insbesondere die Anamnese, Leitlinie des ärztlichen Handelns sein. Eine Gefahr der prädiktiven Diagnostik besteht in der Weckung überzogener Erwartungen bei Arzt und Patient. Molekulare Analytik stellt auch in der Pathologie eine Zusatzuntersuchung dar, die stets am klinischen und pathomorphologischen Bild zu orientieren ist. Darüber hinaus weisen erste Ringversuche auf fehlende Standards in der molekularen Diagnostik hin - mit falsch-negativen und falsch-positiven Befunden ist zu rechnen. Die ethischen und rechtlichen Folgen einer übereilten präventiven «Chirurgie» sind nicht zuletzt auch deshalb kaum abzuschätzen.

Saeger/Pistorius: Zunächst einmal gibt es ethische Grenzen bei einem familiären Tumorleiden, insbesondere bezüglich der pränatalen Diagnostik. Des weiteren haben die molekularen Verfahren natürlich Grenzen hinsichtlich ihrer Sensitivität und Spezifität, und nicht zuletzt ist bei einer Reihe von familiären Tumorerkrankungen die molekulare Ursache bislang nicht bekannt, möglicherweise das entsprechende Gen noch gar nicht charakterisiert.

\section{Frage: Welche Standardverfahren werden derzeit in der molekularen Diagnostik eingesetzt, und wie sieht die zukünftige Entwicklung aus?}

Friedl: Es gibt eine Reihe von Standardverfahren zum Nachweis von bekannten (häufigen) Mutationen (z.B. im RET-Gen) sowie auch zur Suche nach unbekannten Mutationen in verschiedenen Genen. Zu den letzteren gehören Screening-Verfahren, wie z.B. SSCP, Heteroduplexanalyse, PTT, DGGE, TGGE, DHPLC, CCM mit den verschiedenen Varianten die - wegen methodenbedingter Einschränkungen - häufig in Kombination eingesetzt werden. Die Direktsequenzierung genomischer DNA ist wohl die empfindlichste Nachweismethode für Mutationen, die in den entsprechenden PCR-Fragmenten enthalten sind. Es gibt meines Erachtens zur Zeit kein Standardverfahren für die molekulargenetische Diagnostik, das alle Mutationen erfassen kann. Größere Deletionen können z. B. nur mit anderen Methoden (z. B. RT-PCR, Southern-Hybridisierung, quantitative PCR) erfaßt werden. Es ist denkbar, daß in Zukunft die kompletten Sequenzen der genomischen DNA und cDNA für jedes einzelne Gen auf einem «Chip» gespeichert sind, der sowohl qualitative als auch quantitative Veränderungen erken- 
nen kann. In diesem Fall wird der Engpaß nicht mehr bei der Mutationssuche liegen, sondern vielmehr bei der Auswertung und Interpretation der Befunde sowie auch bei der Vermittlung dieser Ergebnisse an die Ratsuchenden und behandelnden Ärzte (humangenetische Beratung, klinische Konsequenzen).

Holinski: Die molekulargenetischen Analyseverfahren werden in Präscreening-Verfahren, mit denen die Position der Mutation eingegrenzt werden kann, und in Verfahren, die einen direkten Mutationsnachweis ermöglichen, eingeteilt. Beide Verfahren haben Vor- und Nachteile. Die Präscreening-Verfahren haben einen hohen Durchsatz, sind kostengünstig, haben aber eine niedrigere Sensitivität als die direkten Verfahren. Ergibt sich bei diesen Methoden ein Hinweis auf eine Mutation, muß der entsprechende Abschnitt des Gens mit einem direkten Nachweisverfahren nachbearbeitet werden. Bei den direkten Verfahren handelt es sich im wesentlichen um die verschiedenen Formen der Sequenzanalyse. Diese Methode hat eine höhere Sensitivität als die Präscreening-Verfahren, sie ist aber wesentlich kostenintensiver und zeitaufwendiger. Zum derzeitigen Zeitpunkt erscheint eine Kombination von Präscreening- und direkten Nachweisverfahren am sinnvollsten. In Zukunft werden automatisierte Chipverfahren, die einen sehr schnellen Mutationsnachweis erlauben, mit funktionellen Analysen kombiniert werden. Funktionelle Analysen sind vor allem bei Mutationen, die zum Aminosäureaustausch führen, wichtig, denn nur eine nachgewiesene Funtionseinschränkung unterscheidet in diesem Fall die Mutation von einer Normvariante.

Möslein: Das Standardverfahren für eine prädiktive Diagnostik besteht in der Sequenzierung und dem direkten Mutationsnachweis. Für eine prädiktive Diagnostik ist der Mutationsnachweis als «Gold-Standard» immer zu fordern. Wünschenswert ist die Bestätigung der krankheitsverursachenden Mutation bei weiteren erkrankten Familienmitgliedern. Inwieweit Präscreening-Verfahren (DGGE, PTT, SSCP etc.) eingesetzt werden oder nicht, ist der Überzeugung und den vorhandenen Ressourcen des jeweiligen Instituts überlassen. Diese Verfahren werden heute vor allem zum Einsparen des Sequenzieraufwandes eingesetzt. Die abzusehende Weiterentwicklung dieser Technik zu einem kostengünstigen, standardisierten Verfahren mit hohem Durchsatz (Chiptechnologie) wird die Präscreening-Verfahren voraussichtlich verzichtbar machen.

Rüschoff: Molekulare Analytik wird künftig wesentlich zur Verbesserung der Krebsfrühdiagnostik, zur Definition von Risikogruppen und zu einer dem Individuum angepaßten (Individual-) Therapie beitragen. Grundsätzlich empfiehlt sich eine stufenweise Methodenkombination. Dies ist für die HNPCC-Diagnostik beispielhaft gelöst: Mikrosatelliten- und immunhistochemische Repair-Genanalyse erfüllen wesentliche Voraussetzungen eines Screening-Tests. Die kostenaufwendige Keimbahnanalyse wird erst danach unter Berücksichtigung der Screening-Daten und der Klinik durchgeführt.
Saeger/Pistorius: Die Palette der gegenwärtig eingesetzten Verfahren ist sehr breit. Sie reicht von relativ simplen Tests auf DNAEbene, wie beispielsweise Mikrosatellitenanalysen, über verschiedene Sceening-Methoden bis hin zur direkten Gensequenzierung oder zu funktionellen Tests auf Proteinebene. Zukünftige Verfahren müssen jedoch noch sensitiver und spezifischer, vor allem aber schneller und kostengünstiger sein. Erste Hinweise für eine entscheidende Erhöhung der Effizienz der direkten Genanalyse kommen insbesondere aus dem Gebiet der sogenannten Chipsequenzierung.

\section{Frage: Welche klinischen Auswirkungen hat die prädiktive molekulare Tumordiagnostik?}

Friedl: Sofern es um die prädiktive Diagnostik von noch gesunden Anlageträgern (ohne Tumor) geht, d. h. um die Darstellung von einer Keimbahnmutation bei der Risikoperson einer Familie mit erblichem Tumorsyndrom, liegt die klinische Relevanz darin, daß man die Anlageträger früher identifizieren kann und ihnen ein entsprechendes Vorsorgeprogramm anbietet. Dadurch kann die Entstehung von Krebs verhindert oder zumindest in einem frühen Stadium erkannt und behandelt werden. Bei den verschiedenen Tumordispositionserkrankungen wurden mehr oder weniger ausgeprägte Beziehungen zwischen Art und Lokalisierung der Mutation im Gen und der klinischen Ausprägung der Erkrankung gefunden. Trotzdem gibt es auch innerhalb der Familien eine breite Streuung bezüglich des klinischen Verlaufs. Daher ist davon auszugehen, daß noch weitere Faktoren (Umweltfaktoren, modifizierende Gene, Zufall) eine Rolle beim Verlauf der Erkrankung spielen. Die Therapie sollte sich deshalb nach dem klinischen Bild bei dem einzelnen Patienten und nicht nur nach der Art und Lokalisierung der Mutation richten.

Möslein: Hier gibt es unterschiedliche Extreme, die von der Penetranz und Heterogenität des Syndroms abhängen. Beispielhaft werden diese Extreme für MEN-II und HNPCC im folgenden erläutert: Bei MEN-II ergibt sich allein aus dem Mutationsnachweis im RET-Protoonkogen die klare Indikation zu einer prophylaktischen Thyreoidektomie im Kindesalter. Man wartet nicht ab, bis biochemische Markerveränderungen auftreten oder gar makroskopisch sichtbare Organveränderungen nachweisbar sind. Bislang wurde bei allen in Deutschland prophylaktisch mit dieser Indikation thyreoidektomierten Personen zumindest eine C-Zell-Hyperplasie oder ein Karzinom nachgewiesen.

Bei HNPCC hingegen ist aufgrund der erheblichen auch intrafamiliären Heterogenität der Krankheitsausprägung die Entfernung eines Organs ohne neoplastische Veränderungen sehr zurückhaltend zu stellen. Die Penetranz einer Mutation in einem der Mismatchrepair-Gene erreicht etwa $80 \%$. Diese Penetranz bezieht sich allerdings auf alle Organsysteme. Es stellt sich somit die Frage, welches Organ zu welchem Zeitpunkt prophylaktisch entfernt werden sollte. Darüber hinaus sind nicht nur verzichtbare Organe wie Kolon und Endometrium oder Magen betroffen, sondern auch 
Pankreas, ableitende Harnwege, Dünndarm, Ovarien etc. Die extreme Gefährdung der Entwicklung syn- oder metachroner Kolonkarzinome ist andererseits bei HNPCC eindeutig belegt. Es scheint Genträger zu geben, die eine sehr hohe Penetranz haben, kolorektale Karzinome auszubilden. Diese treten auch in kurzen Intervallen auf, manchmal ohne Ausbildung einer Adenom-Karzinom-Sequenz als De novo-Karzinome. Bei Personen, die ein neoplastisches Potential durch multiple Adenome oder ein kolorektales Karzinom aufweisen, sollte ein präventiv-chirurgischer Eingriff durchaus nahegelegt werden. Bei einem Kolonkarzinom ist hier die Ausweitung des Eingriffs im Sinnne einer subtotalen Kolektomie mit ileorektaler Anastomose zu empfehlen. Bei einem primären Rektumkarzinom ist sogar eine restaurative Proktokolektomie mit ileoanaler Pouch-Anlage zu diskutieren. Ob eine regelmäßige Vorsorge durch Koloskopien mit konsequenter Polypabtragung einen vergleichbaren präventiv-medizinischen Effekt erzielen kann wie eine prophylaktische Chirurgie, muß noch untersucht werden.

Rüschoff: Die prädiktive Diagnostik wird zu einer wesentlichen Verbesserung der Patientenversorgung, insbesondere des Tumorpatienten, führen. Wir kommen zu einer risikoadaptierten, individualisierten und damit auch zu einer besser finanzierbaren Therapie. Gleichzeitig werden durch diesen Ansatz die medizinischen Disziplinen enger zusammenarbeiten (müssen) und damit wird auch die Pathologie näher an das Krankenbett rükken.

Saeger/Pistorius: Durch die bereits erwähnte mögliche klare Differenzierung von Genträgern und Nichtgenträgern in Familien, in denen die pathogenetische Keimbahnmutation identifiziert werden konnte, ist es möglich, gezielte und individuell spezifische klinische Vorsorgeprogramme zu etablieren. Wir versprechen uns davon eine Verbesserung der Prognose, da die Neoplasien in einem sehr frühen, oft noch präklinischen Stadium erkannt und therapiert werden können. Außerdem ist es durch die Kenntnis der spezifischen Tumorbiologie möglich, bei einem Teil dieser Patienten das operative Vorgehen der jeweiligen Konstellation anzupassen und somit ebenfalls eine Prognoseverbesserung zu erzielen. Möglicherweise können zukünftig auch effektive medikamentöse Präventivmaßnahmen bei molekulargenetisch charakterisierten $\mathrm{Pa}$ tienten eingesetzt werden. Für diejenigen, die innerhalb einer solchen Familie als Nichtgenträger identifiziert werden, bedeutet das Ergebnis der molekularen Diagnostik eine Entlastung; ihnen können weitere regelmäßige, nicht selten invasive Untersuchungen erspart werden.

\section{Frage: Was sind die ethischen Aspekte der prädik- tiven molekularen Diagnostik?}

Irrgang: Aus der Perspektive einer medizinischen Ethik, die von der Patientenautonomie ausgeht, möchte ich folgende Thesen formulieren: 1. Es gibt ein Recht des potentiell Betroffenen zu ent- scheiden, ob er Kenntnis von einer genetischen Prädisposition haben möchte. 2. Prädiktive molekulare Diagnostik sollte angewandt werden, wenn es sich um behandelbare Krankheiten handelt. 3. Bei nichtbehandelbaren genetisch bedingten Erkrankungen gibt es ein uneingeschränktes Recht auf Nichtwissen. 4. Bei Auswirkungen auf andere (z.B. auf eigene Nachkommen) wird dieses Recht durch Fürsorgepflichten für diese eingeschränkt. 5. Bei der Mitteilung von schweren nichtbehandelbaren Erkrankungsrisiken hoher Wahrscheinlichkeit ist die Mitteilung der Diagnose psychologisch/psychotherapeutisch zu begleiten. 6. Ergebnisse einer Diagnose im Rahmen der prädiktiven Medizin dürfen nur an den Betroffenen weitergegeben werden, um die Gefahr diskriminierender oder vielleicht gar selektionistischer gesellschaftlicher Wünsche gar nicht erst aufkommen zu lassen. 7. Der Arzt trägt die Verantwortung für eine möglichst genaue Diagnose und für Hinweise auf (noch) bestehende Grenzen im Prognoseverfahren selbst.

Der Sinn von Diagnoseverfahren ohne jede therapeutisch-präventive Konsequenzen kann aus ethischer Perspektive bezweifelt werden. Präventive Therapieformen für bestehende Diagnoseverfahren sind bevorzugt zu entwickeln.

Friedl: Bei der prädiktiven molekulargenetischen Diagnostik wird bei einer noch gesunden Person die Diagnose einer erhöhten Tumordisposition gestellt für ein Tumorsyndrom, das in der Familie bereits bekannt ist. Die ethischen Aspekte, die damit verbunden sind, werden auch in den Richtlinien zur Diagnostik der genetischen Disposition für Krebserkrankungen der Bundesärztekammer [Dtsch Ärzteblatt 1998;95:1396-1403] ausführlich dargestellt. In diesem Sinne ist das von der Deutschen Krebshilfe geförderte Pilotprojekt zur molekulargenetischen Diagnostik beim familiären Brustkrebs sehr zu begrüßen. Diese Projekte haben zum Ziel, die molekulare Diagnostik zu etablieren und deren klinisch-präventive Relevanz, aber auch die psychosozialen Konsequenzen dieser Diagnostik zu eruieren, bevor über eine Einführung dieser Diagnostik in die klinische Praxis entschieden wird.

Holinski: Wenn in einer belasteten Familie die krankheitsverursachende Mutation bekannt ist, wird in manchen Fällen die genetische Testung der Kinder gewünscht. Besteht nach derzeitigem Wissen im jeweiligen Fall im Kindes- und Jugendalter kein erhöhtes Erkrankungsrisiko, stehen wir diesem Wunsch eher ablehnend gegenüber. Zum einen hat jeder Mensch nach den internationalen Richtlinien ein Recht auf Nichtwissen, das gewahrt werden sollte. Kinder sollten daher nach Vollendung des 18. Lebensjahres selbst entscheiden, ob sie eine prädiktive genetische Diagnostik wünschen. Zum anderen wird das Wissen um eine genetische Belastung des Kindes dessen Erziehung beeinflussen, auch wenn die Eltern sich bemühen, dies zu vermeiden. Eine pränatale genetische Testung zum Zwecke der Risikoeinschätzung des ungeborenen Kindes erscheint nicht vertretbar, da die meisten familiär auftretenden Tumorerkrankungen sich im Erwachsenenalter manifestieren und bis dahin voraussichtlich bessere Früherkennungs- und Therapieprogramme zur Verfügung stehen werden. 
Möslein: Bei der «prädiktiven Diagnostik» geht es um eine neue Dimension in der Medizin, die sich durch technische Fortschritte eröffnet hat. Die durch diese Technik möglich gewordene Vorhersage einer bestimmten Krankheit oder anderer «Defekte», bevor diese klinisch manifest sind, hat potentiell Auswirkungen auf die individuelle Lebenssituation, das zukünftige Verhalten und möglicherweise die kompletten Lebensentwürfe. Die Kenntnis eines Genträgerstatus wird nicht nur Konsequenzen für die molekulargenetisch untersuchte Person, sondern zugleich für die lebenden Verwandten und die eventuellen Nachkommen haben. Mit Auswirkungen auf das Arzt-Patient-Verhältnis, die Familienplanung, Arbeits- und Versicherungsverhältnisse und nicht zuletzt das Gesundheitssystem ist zu rechnen. Es werden zwei ethische Leitmotive in der Medizin berührt: der Nutzen des Patienten und die Verantwortung des Arztes. Es gilt nun, die bioethische Diskussion über diese neuartigen Situationen einer prädiktiven Medizin zu eröffnen und die ethischen Werte zu definieren. Die vorhandenen ethischen Standards müssen Voraussetzungen schaffen, um das präventive Potential unter den Vorzeichen der Selbstbestimmung und der Autonomie maximal einsetzen zu können. Stets muß die Vertraulichkeit der Informationen gewahrt bleiben, um vor sozialer Diskriminierung und Mißbrauch z. B. durch Versicherungen zu schützen. Ob, wie in den angelsächsischen Ländern diskutiert, ein «genetic privacy law» erforderlich ist oder ob andere Modelle umsetzbar sind, die die Kernbereiche der Privatsphäre des Menschen schützen, muß in einer öffentlich geführten Diskussion entschieden werden.

Rüschoff: Eine grundsätzliche Gefahr besteht darin, daß Analysen durchgeführt werden, deren klinische Relevanz einerseits und deren psychische Folgen andererseits kaum (oder gar nicht) durch prospektive Studien abgesichert sind. Gleichzeitig ist das Gebiet so neu, daß es auch in der Ärzteschaft noch an breiter Erfahrung in der Beratung von Patienten z. B. mit molekular gesichertem erblichem Dickdarmkrebs fehlt. Auf diesem Hintergrund sollte Kritikfähigkeit unser Handeln bestimmen. Die ethischen Folgen sind derzeitig kaum abzuschätzen.

Saeger/Pistorius: Auf dem Gebiet der hereditären Tumorerkrankungen sollte unseres Erachtens die pränatale molekulare Diagnostik nicht eingesetzt werden. Die entsprechenden Patienten bzw. Risikopersonen müssen intellektuell in der Lage sein, sich nach entsprechender Aufklärung für oder gegen diese Untersuchungen zu entscheiden. Die Situation ist hier ähnlich wie vor einem operativen Eingriff. Nur von volljährigen Patienten wird hier eine selbständige Entscheidungskompetenz erwartet. Andererseits geht die Einwilligung zur molekulargenetischen Untersuchung weit über die für einen operativen Eingriff hinaus. Im Falle des Nachweises einer pathogenetischen Keimbahnmutation kann die psychische Belastung für den Patienten erheblich sein. Dies gilt besonders dann, wenn sich aus dem Ergebnis der Untersuchung keine unmittelbaren therapeutischen Schritte ableiten lassen. Es ist deshalb unabdingbar, daß die Beratung von potentiellen Mutationsträgern durch ausgesprochen erfahrene Ärzte unter Einbeziehung von $\mathrm{Hu}$ - mangenetikern und Psychologen erfolgt. Insgesamt muß der Nutzen der Diagnoseerstellung für den Patienten und seine Angehörigen eindeutig erkennbar sein.

\section{Frage: Gibt es auch genetische Risikofaktoren für sporadische Tumoren und welche Bedeutung könnte dies für die Prädiktion und Prävention haben?}

Friedl: «Sporadische» Tumoren beruhen definitionsgemäß nicht auf einer «autosomal dominant vererbten» Tumordisposition im Sinne der oben beschriebenen Tumorsyndrome. Tatsächlich beruht jeder Tumor - auch der «sporadische» - auf einem Fehler in der Kontrolle des Zellwachstums. Im Zellzyklus werden verschiedene Regulatoren zu ganz bestimmten Zeiten an- oder abgeschaltet, und die einzelnen Komponenten sind zeit- und mengenmäßig sehr exakt aufeinander abgestimmt. Es ist daher sehr wahrscheinlich, daß minimale funktionelle Unterschiede, die genetisch bedingt sein können, in einem dieser Faktoren noch keine erhöhte Tumordisposition auslösen, eine bestimmte Kombination von funktionellen Varianten oder auch zusätzlichen Umweltfaktoren kann jedoch den Regelkreis erheblich modulieren. Man spricht dann von polygenen Faktoren (multifaktoriellen Auswirkungen, genetischem Hintergrund), die eine Tumordisposition erhöhen oder vermindern. In der Regel ist der Einfluß jedes einzelnen Faktors relativ gering und kann nur an einer größeren Zahl von Personen ermittelt werden. Für den einzelnen haben diese genetischen Risikofaktoren für sporadische Tumoren - nach heutigem Stand der Erkenntnis - keine praktische Relevanz für Prädiktion und Prävention. Deren Untersuchung in Forschungsprojekten kann jedoch einen wichtigen Beitrag zur weiteren Aufklärung des komplexen Prozesses der Tumorentstehung und -progression leisten.

Holinski: An der Erforschung genetischer Risikofaktoren für sporadische Tumorerkrankungen wird gearbeitet. Bisher gibt es keine sicheren Konzepte, die prädiktiv oder präventiv einsetzbar wären. $\mathrm{Ob}$ es sichere Konzepte geben wird und wie diese anzuwenden sind, kann nicht vorhergesagt werden.

Möslein: Niedrig-penetrante genetische Risikofaktoren könnten bei zahlreichen «sporadischen» Tumoren eine Rolle spielen. Es ist damit zu rechnen, daß auch diese Faktoren in nächster Zukunft analysiert und quantifiziert werden können. Vorstellbar ist, daß aufgrund dieser molekulargenetischen Risikoanalyse ein klinisch umsetzbares individuelles Erkrankungsprofil erstellt werden kann. Die Bedeutung präventiver Operationsindikationen wird voraussichtlich in naher Zukunft zunehmen.

Rüschoff: Die Forschung über die Bedeutung sogenannter Modifier-Gene für die Entstehung «sporadischer» Tumoren ist derzeitig noch in den Kinderschuhen. Es ist allerdings zu erwarten, daß dies für die Erkennung von Risikogruppen künftig von erheblicher epidemiologischer und gesundheitspolitischer Bedeutung sein wird. 
Saeger/Pistorius Wenn wir davon ausgehen, daß Tumoren als Resultat der Wirkung von endogenen und exogenen Faktoren, wenn auch in unterschiedlicher Gewichtung, entstehen, so wäre es möglich, durch die Identifizierung von endogenen, d. h. genetischen Risikofaktoren innerhalb der Vielzahl von Patienten Risikogruppen zu definieren. Den entsprechenden Patienten könnte dann präzise die Meidung von exogenen Risikofaktoren, beispielweise bei der
Ernährung, empfohlen werden. Vorsorgeuntersuchungen könnten individuell programmiert und eine medikamentöse Prävention sehr gezielt eingesetzt werden. Erste Hinweise für das Vorliegen solcher genetischer Risikofaktoren für sporadische Tumoren gibt es bereits beim kolorektalen Karzinom; weitere werden sicher folgen.

\section{Teilnehmer}

Dr. Waltraut Friedl

Institut für Humangenetik

Rheinische Friedrich-Wilhelms-Universität

Wilhelmstraße 31

D-53111 Bonn

Dr. Elke Holinski-Feder

Abteilung Medizinische Genetik

Ludwig-Maximilians-Universität

Goethestraße 29

D-80336 München

Prof. Dr. Dr. B. Irrgang

Institut für Philosophie

Technische Universität Dresden

Mommsenstraße 13

D-01062 Dresden
PD Dr. Gabriela Möslein

Chirurgische Klinik und Poliklinik

Heinrich-Heine-Universität

Postfach 101007

D-40001 Düsseldorf

Dr. St. Pistorius

Klinik für Vizeral-, Thorax- und Gefäßchirurgie

Technische Universität Dresden

Fetscherstraße 74

D-01307 Dresden

Prof. Dr. J. Rüschoff

Institut für Pathologie

Städtische Kliniken Kassel

Mönchebergstraße 41-43

D-34125 Kassel
Prof. Dr. H.-D. Saeger

Klinik für Vizeral-, Thorax- und Gefäßchirurgie

Technische Universität Dresden

Fetscherstraße 74

D-01307 Dresden 